\title{
Sex Hormones and Sleep in Men and Women from the General Population: A Cross-Sectional Observational Study
}

Hanna Kische1 ${ }^{1}$, Ralf Ewert², Ingo Fietze ${ }^{3}$, Stefan Gross ${ }^{4,5}$, Henri Wallaschofski ${ }^{1,4}$, Henry Völzke 4,6 , Marcus Dörr ${ }^{4,5}$, Matthias Nauck ${ }^{1,4}$, Anne Obst ${ }^{2,6}$, Beate Stubbe ${ }^{2}$, Thomas Penzel ${ }^{3}$, and Robin Haring ${ }^{1,7}$

${ }^{1}$ Institute of Clinical Chemistry and Laboratory Medicine, ${ }^{2}$ Department of Internal Medicine B, University Medicine Greifswald, Germany, ${ }^{3}$ Interdisciplinary Sleep Center, Charité,

University Medicine Berlin, Germany, ${ }^{4}$ German Centre for Cardiovascular Research (DZHK), partner site Greifswald, Germany, ${ }^{5}$ Department of Cardiology, ${ }^{6}$ Institute for

Community Medicine, University Medicine Greifswald, Germany, ${ }^{7}$ European University of Applied Sciences, Faculty of Applied Public Health, Rostock, Germany

Background Sleep is a complex vital state and a highly active and dynamic process occupying one third of human's lifetime. The prevalence of insomnia worldwide is estimated to be $10-30 \%$ and insomnia is associated with a higher risk of hypertension, obesity, metabolic syndrome and depression (2). To date, associations between sex hormones and sleep were mainly investigated in connection with hormone replacement therapy (1), menopausal status (4) and menstrual cycle (5) in women and obstructive sleep apnoea in men (6). Thus, we examined a comprehensive panel of sex hormones and various sleep characteristics in a population-based sample of healthy men and women from the general population.

\section{METHODS}

- We used data from 204 men and 213 women of the Study of Health in Pomerania TREND, who underwent cardiorespiratory polysomnography.

- Associations of total and free testosterone ( $T$, $f T$ ), androstenedione (ASD), estrone (E1), and estradiol (E2) measured by liquid chromatography-tandem mass spectrometry, and sex hormone-binding globulin (SHBG), dehydroepiandrosterone-sulphate (DHEAS) and E2/TT ratio with sleep measures were investigated.

- Sleep measures include total sleep time (TST), sleep efficiency, wake after sleep onset (WASO), apnea-hypopnea index (AHI), Insomnia severity index (ISS), Epworth sleepiness scale (ESS), and Pittsburgh sleep quality index (PSQI).

- Sex-specific multivariable regression models were adjusted for age, waist circumference, hypertension, smoking, physical inactivity, and alcohol consumption. Sensitivity analyses were performed with stratification by diagnosis of depression and menopausal status (women).

\section{RESULTS}

- In men, associations of $\Pi$, $\mathrm{fT}, \mathrm{SHBG}$, and E2/TT ratio with AHI in age-adjusted analyses were rendered non-significant after multivariable adjustment

(Figure 1A, Table 1). In multivariable analyses, ASD was associated with ESS (Table 1).

- In women, only age-adjusted models showed an inverse association of SHBG with AHI (Figure 1B, Table 1).

Multivariable analyses showed positive associations of DHEAS with WASO and of E2 and E2/TT ratio with ESS (Figure 1C, Table 1).

- Additionally, $\mathrm{fT}$ and SHBG were associated with AHI in multivariable models in pre-menopausal women.

A) AHI by SHBG in men

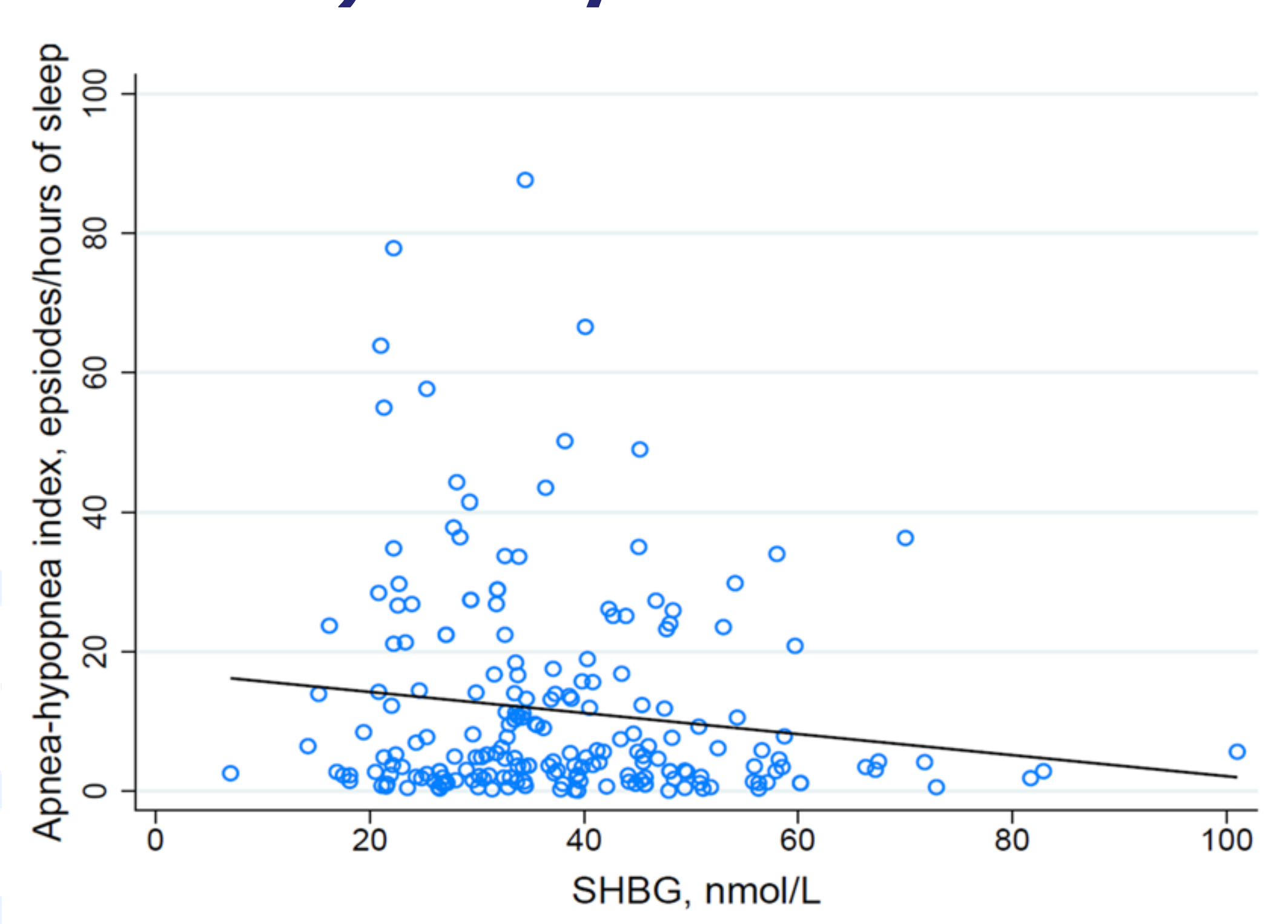

Figure 1: Scatterplot of

B) AHI by SHBG in women

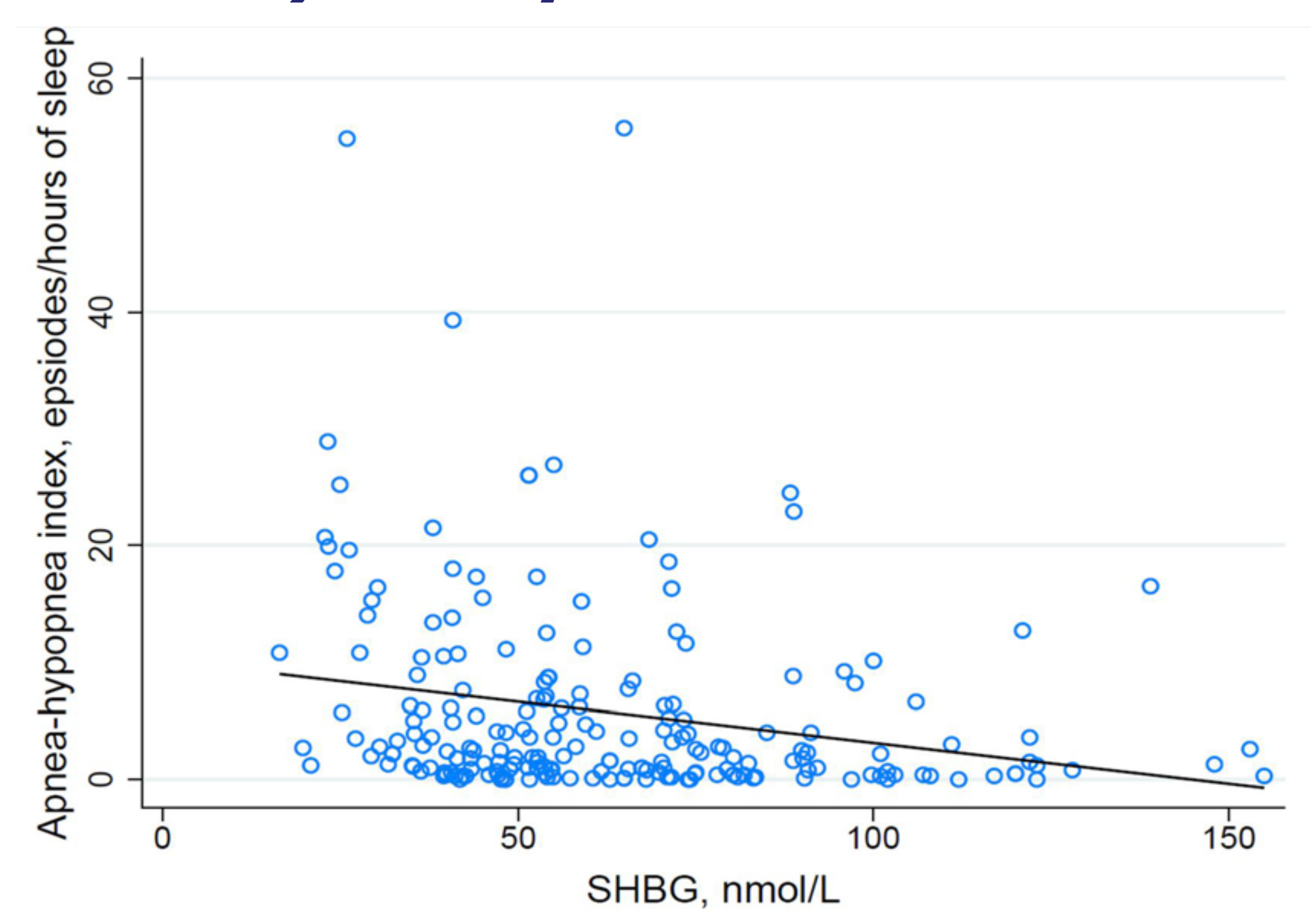

C) ESS by E2 in women

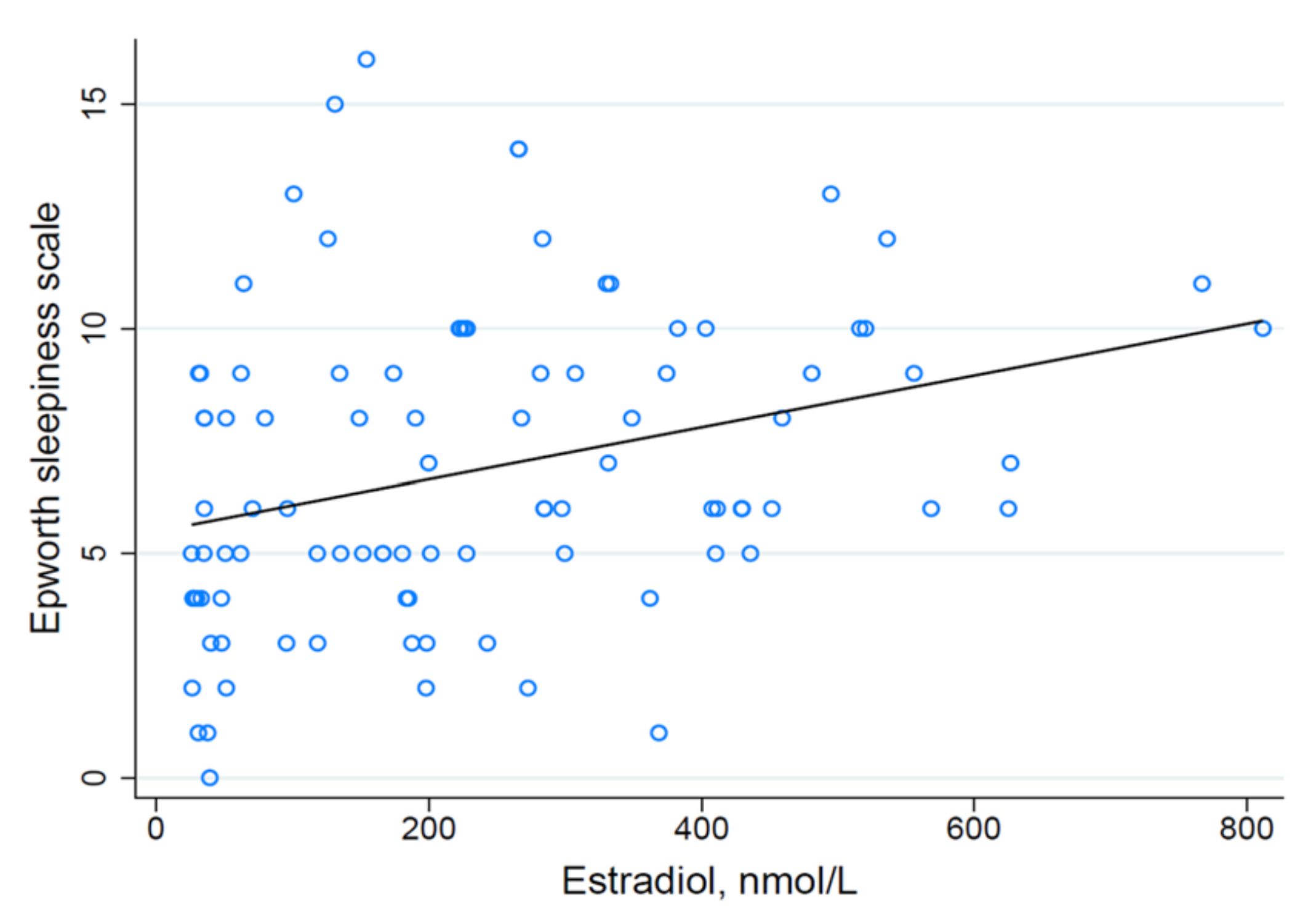

Table 1: Sex-specific, cross-sectional associations of sex hormones and SHBG with sleep measures in linear regression models.

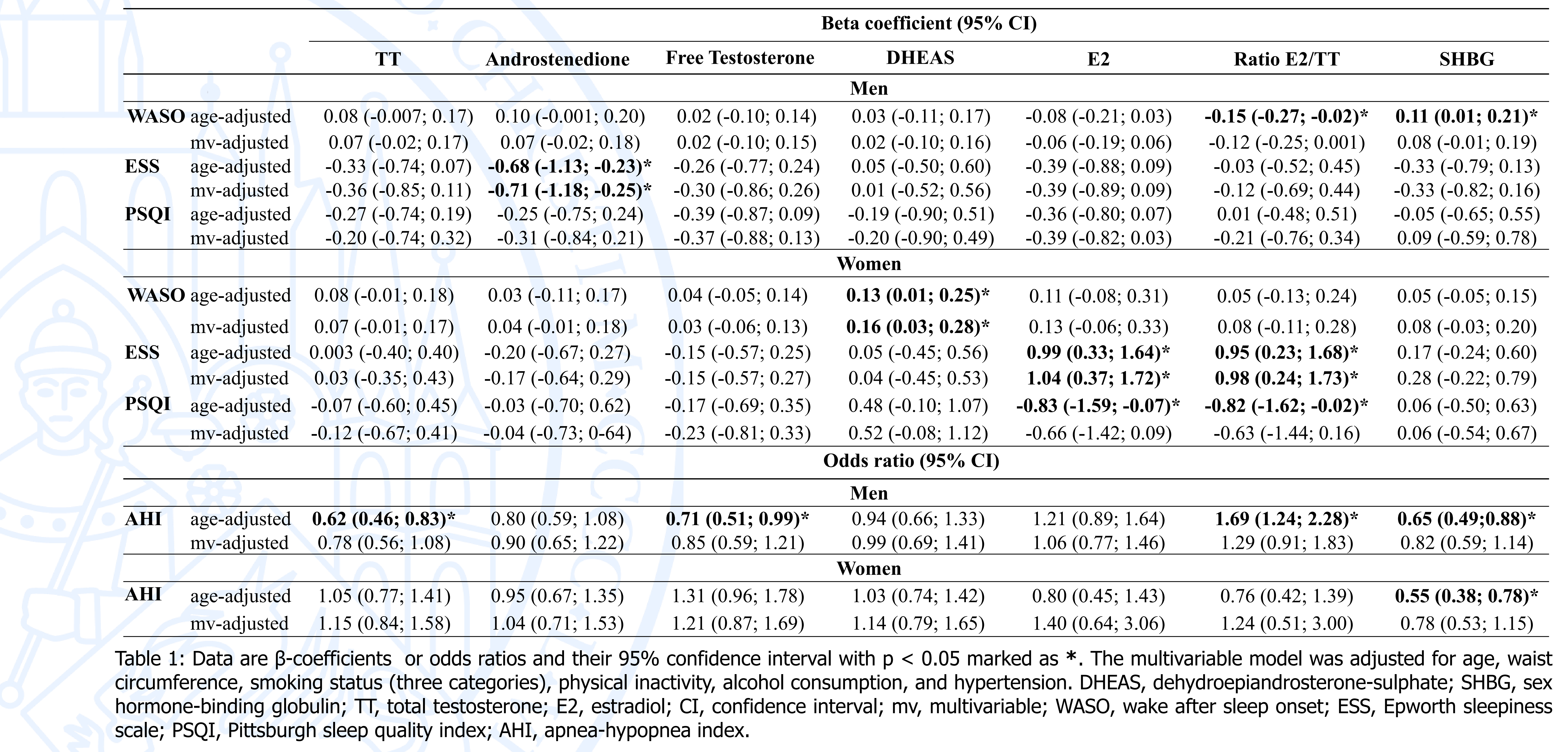

Conclusion Consistent with previous findings, the present cross-sectional population-based study observed sex-specific associations of testosterone, SHBG, and estrogen with sleep disturbances and poor sleep quality. However, multivariable-adjusted analyses confirmed the relative impact of body composition, health-related lifestyle, and comorbidity on the association between sex hormones and sleep. 